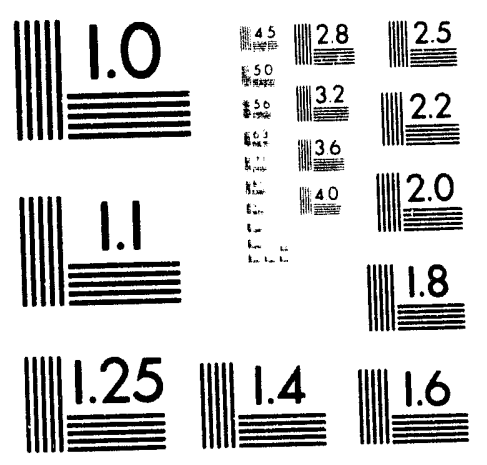



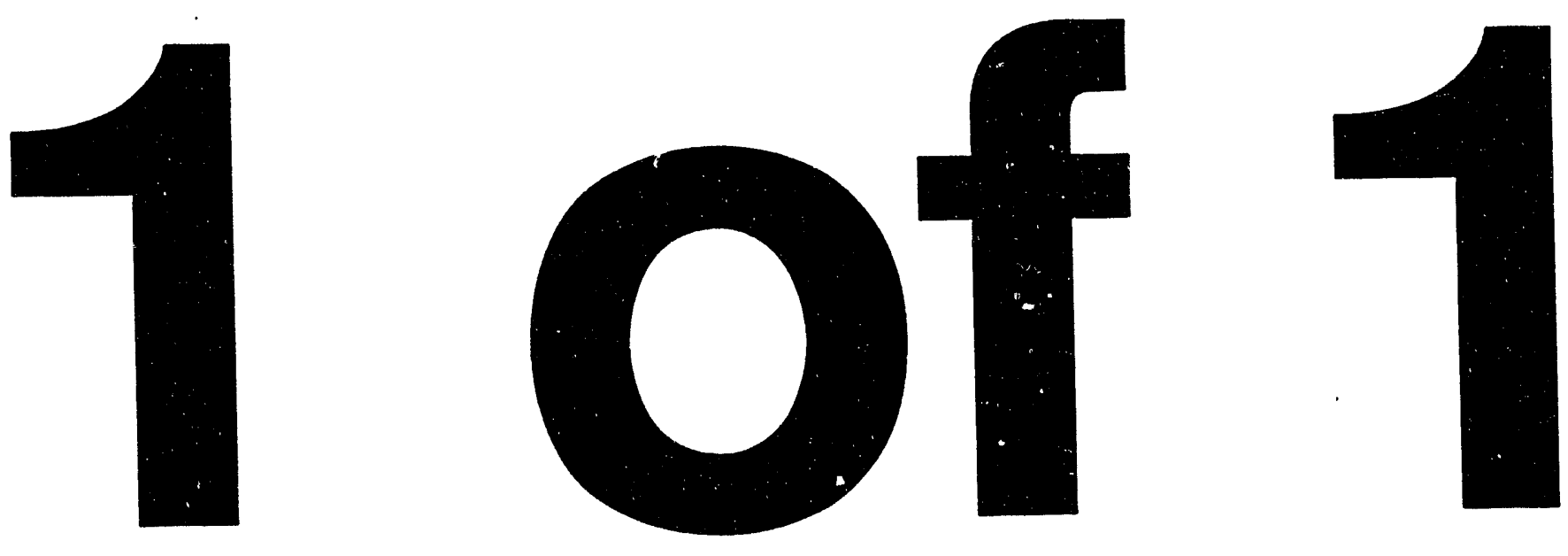


\title{
Laser Propagation in Underdense Plasmas: Scaling Arguments
}

\author{
John C. Garrison
}

May 1993

This is an informal report intended primarily for internal or limited external distribution. The opinions and conclusions stated are those of the author and may or may not be those of the Laboratory.

Work performed under the auspices of the U.S. Department of Energy by the Lawrence Livermore National Laboratory under Contract W-7405-Eng-48. 


\section{DISCLAIMER}

This document was prepared as an acccount of work sponsored by an agency of the United States Government. Neither the United States Government nor the University of California nor any of their employees, makes any warranty, express or implied, or assumes any legal liability or responsibility for the accuracy, completeness, or usefulness of any information, apparatus, product, or process disclosed, or represents that its use would not infringe privately own rights. Reference herein to any specific commercial products, process, or service by trade name, trademark, manufacturer, or otherwise, does not necessarily constitute or imply its endorsement, recommendation, or favoring by the United States Government or the University of California. The views and opinions of authors expressed herein do not necessarily state or reflect those of the United States Government or the University of California, and shall not be used for advertising or product endorsement purposes.

This report has been reproduced directly from the best available copy.

$$
\begin{gathered}
\text { Available to DOE and DOE contractors from the } \\
\text { Office of Scientific and Technical Information } \\
\text { P.O. Box 62, Oak Ridge, TN } 37831
\end{gathered}
$$

Prices available from (615) 576-8401, FTS 626-8401

Available to the public from the

National Technical Information Service

U.S. Department of Commerce

5285 Port Royal Rd.

Springfield, VA 22161 


\title{
Laser Propagation in Underdense Plasmas: Scaling Arguments
}

\author{
John C. Garrison
}

\begin{abstract}
The propagation of an intense laser beam in an underdense plasma is modelled by treating the plasma as a relativistic, zero temperature, charged fluid. For paraxial propagation and a sufficiently underdense plasma $\left(\omega_{\mathrm{p}} / \omega<1\right)$, a multiple-scales technique is used to expand the exact equations in powers of the small parameter $\theta \equiv \omega_{\mathrm{p}} / \omega$. The zeroth order equations are used in a critical examination of previous work on this problem, and to derive a scaling law for the threshold power required for cavitation.
\end{abstract}




\section{Introducuon}

The interaction of high intensity laser pulses with matter inevitably produces plasmas; therefore a natural first step in the investigation of intense laser-matter interactions is the study of propagation of intense fields in preformed plasmas. For underdense plasmas (plasma frequency less than the laser frequency), the simplest model is provided by a magnetohydrodynamic (MHD) desc intion of the plasma. A further simplification comes fromi the observation that the pulse durations required to produce high intensities are so short that no appreciable motion of the ions can take place during the pulsa. This allows a one-fluid description of the plasma in which the ions are represented by a stationary continuous positive charge distribution, while the electrons are modelled by a continuous negative charge distribution which responds dynamically to the injected laser pulse. The very intense fields of interest in this problem will drive the electrons to relativistic velocities in one optical period; therefore the initial nonrelativistic velocities of the plasma electrons can be neglected, and the electrons can be treated as a zero temperature fluid. It is important to keep in tilind that the MHD model is based on the assumption that the laser pulse only interacts with the collective degrees of freedom represented by the fluid density and velocity, i.e., that the laser field is not strongly coupled to the microscopic particle degrees of freedom of the plasma. Since this assumption has not been independently verified, the MHD model for propagation should be complemented by particle simulations. The present report is primarily intended to explain the basic MHD theory and present some important scaling considerations in more detail than is usually found in the literature.

The total electromagnetic field in the plasma is described by the vector and scalar potentials $\mathbf{A}$ and $\Phi$. In the radiation gauge $(\nabla \cdot \mathbf{A}=0)$ the vector potential satisfies the wave equation [1]

$$
\left(\nabla^{2}-\frac{1}{c^{2}} \frac{\partial^{2}}{\partial t^{2}}\right) \mathbf{A}=-\frac{4 \pi}{c} \mathbf{J}+\frac{1}{c} \nabla \frac{\partial \Phi}{\partial t},
$$

and the scalar potential satisfies Poisson's equation

$$
\nabla^{2} \Phi=-4 \pi \rho
$$

The charge and current densities of the MHD model are given by

$$
\rho(r, t)=-e N(r, t)+e N_{0} f(r),
$$




$$
J(r, t)=-e N(r, t) v(r, t)
$$

where $N$ and $v$ are respectively the electron density and velocity, and $N_{0} f(r)$ is the positive background density. The dimensionless function $f(r)$ satisfies $0 \leq f(r) \leq 1$, so $N_{0}$ is the maximum background density. In the undisturbed plasma there is local charge neutrality so that $N(r,-\infty)=N_{0} f(r)$. The vector potential in (1.1) represents the total electromagnetic field. We will see below that the total field is expressed by $\mathbf{A}=\mathbf{A}_{\mathbf{L}}+\mathbf{A}_{\mathbf{w}}$ where $\mathbf{A}_{\mathbf{L}}$ represents the laser field and $\mathbf{A}_{\mathbf{w}}$ represents the wake field generated by plasma excitations.

The response of the electron fluid to the total field is described by the continuity equation

$$
\frac{\partial N}{\partial t}+\nabla \cdot(N \mathbf{v})=0
$$

and the Lorentz force law

$$
\frac{\mathrm{DP}}{\mathrm{D} x}=-\mathbf{e} \mathbf{E}-\frac{\mathrm{e}}{\mathrm{c}} \mathbf{v} \times \mathbf{B}
$$

where the material derivative, the electric field, the magnetic field, and the electron momentum are given by

$$
\begin{aligned}
& \frac{\mathrm{D}}{\mathrm{D} t} \equiv \frac{\partial}{\partial \mathrm{t}}+\mathbf{v} \cdot \nabla, \\
& \mathbf{E}=-\nabla \Phi-\frac{1}{\mathrm{c}} \frac{\partial \mathrm{A}}{\partial \mathrm{t}}, \quad \mathbf{B}=\nabla \times \mathbf{A}, \\
& \mathbf{P}=\mathrm{m}_{0} \gamma \mathbf{v}, \quad \gamma=\frac{1}{\sqrt{1-\frac{\mathrm{v}^{2}}{\mathrm{c}^{2}}}} .
\end{aligned}
$$

The equation of motion (1.5) can be expressed directly in terms of the vector potential by writing it out as

$$
\frac{\partial \mathbf{P}}{\partial \mathrm{t}}+(\mathbf{v} \cdot \nabla) \mathbf{P}=\mathrm{e} \nabla \boldsymbol{\Phi}+\frac{\mathrm{e}}{\mathrm{c}} \frac{\partial \mathbf{A}}{\partial \mathrm{t}}-\frac{\mathrm{e}}{\mathrm{c}} \mathrm{v} \times(\nabla \times \mathbf{A})
$$

and using the identity $\mathbf{S} \times(\nabla \times \mathbf{T})=\mathrm{S}_{\mathrm{j}} \nabla \mathrm{T}_{\mathrm{j}}-(\mathbf{S} \cdot \nabla) \mathbf{T}$ to set 


$$
(\mathbf{v} \cdot \nabla) \mathbf{P}=\mathbf{v}_{\mathbf{j}} \nabla \mathrm{P}_{\mathrm{j}}-\mathbf{v} \times(\nabla \times \mathbf{P})
$$

The first term on the right in (1.8) can be expressed by

$$
\begin{aligned}
& \mathrm{v}_{\mathrm{j}} \nabla \mathrm{P}_{\mathrm{j}}=\mathrm{m}_{0} \mathrm{v}_{\mathrm{j}}\left(\nabla \gamma \mathrm{v}_{\mathrm{j}}+\gamma \nabla \mathrm{v}_{\mathrm{j}}\right)=\mathrm{m}_{0}\left(\nabla \gamma \mathrm{v}^{2}+\frac{1}{2} \gamma \nabla \mathrm{v}^{2}\right), \\
& \mathrm{v}^{2}=\mathrm{c}^{2}\left(1-\frac{1}{\gamma^{2}}\right) \Rightarrow \nabla \mathrm{v}^{2}=\frac{2 c^{2}}{\gamma^{3}} \nabla \gamma, \\
& \Rightarrow \mathrm{v}_{\mathrm{j}} \nabla \mathrm{P}_{\mathrm{j}}=\mathrm{m}_{0} \mathrm{c}^{2} \nabla \gamma,
\end{aligned}
$$

and this changes (1.8) to

$$
(\mathbf{v} \cdot \nabla) \mathbf{P}=\mathrm{m}_{0} c^{2} \nabla \gamma-\mathrm{v} \times(\nabla \times \mathbf{P})
$$

Putting this in (1.7) yields an equivalent form of the Lorentz force law,

$$
\frac{\partial}{\partial t}\left(\mathbf{P}-\frac{\mathrm{e}}{\mathrm{c}} \mathbf{A}\right)-\mathbf{v} \times\left[\nabla \times\left(\mathbf{P} \frac{\mathrm{e}}{\mathrm{c}} \mathbf{A}\right)\right]=\mathrm{e} \nabla \Phi-\mathrm{m}_{0} \mathrm{c}^{2} \nabla \gamma,
$$

which can be further simplified by the following considerations. Let $\Omega \equiv \nabla \times\left(\mathbf{P}-\frac{\mathrm{e}}{\mathrm{c}} \mathbf{A}\right)$ and take the curl of (1.10) to get

$$
\frac{\partial}{\partial t} \Omega-\nabla \times(v \times \Omega)=0,
$$

which is a first order, linear, and homogeneous differential equation for $\Omega$. Consequently, if $\Omega\left(r, t_{0}\right)=0$ at some initial time $t_{0}$, then $\Omega(r, t)=0 \forall t>t_{0}$. The initial conditions of interest are that the plasma is quiescent and that the field $A$ vanishes; consequently $\Omega(r, t)=0 \forall t>t_{0}$. The force law (1.10) can then be simplified to

$$
\frac{\partial}{\partial t}\left(\mathbf{P}-\frac{\mathrm{e}}{\mathrm{c}} \mathbf{A}\right)=\mathrm{e} \nabla \Phi-\mathrm{m}_{0} \mathrm{c}^{2} \nabla \gamma
$$

together with the initial condition

$$
\nabla \times\left.\left(\mathbf{P}-\frac{e}{c} \mathbf{A}\right)\right|_{t=t_{0}}=0 .
$$


It is useful to describe the strong fields and relativistic motions in this problem by introducing normalized variables

$$
\begin{aligned}
& a \equiv \frac{e A}{m_{0} c^{2}}, \phi \equiv \frac{e \Phi}{m_{0} c^{2}}, \\
& p \equiv \frac{p}{m_{0} c}, \quad \frac{v}{c} \equiv \frac{p}{\gamma} \quad \gamma=\sqrt{1+p^{2}}
\end{aligned}
$$

The electron density $\mathrm{N}$ is measured in the laboratory frame, but it is more convenient to use $\mathrm{N} / \gamma$, the electron density in the local fluid rest frame. The normalized electron density will therefore be represented by

$$
\eta \equiv \frac{\mathrm{N}}{\gamma \mathrm{N}_{0}}
$$

The normalized equations are then

$$
\begin{aligned}
& \nabla \cdot a=0, \\
& \left(\nabla^{2}-\frac{1}{c^{2}} \frac{\partial^{2}}{\partial t^{2}}\right) a=k_{P}^{2} \eta \mathbf{p}+\frac{1}{c} \nabla \frac{\partial \phi}{\partial t}, \\
& \nabla^{2} \phi=k_{p}^{2}(\gamma \eta-f), \\
& \frac{1 \partial}{c \partial t}(p-a)=\nabla(\phi-\gamma), \\
& \frac{1 \partial}{c \partial t}(\eta \gamma)+\nabla \cdot(\eta \mathbf{p})=0,
\end{aligned}
$$

where $k_{p}=\omega_{p} / c$ and $\omega_{p}$ is the standard (nonrelativistic) plasma frequency: $\omega_{\mathrm{p}}^{2}=4 \pi \mathrm{N}_{0} \mathrm{e}^{2} / \mathrm{m}$. 


\section{Multiple scales expansion}

\section{A Basic scaling arguments.}

The normalized equations (1.14) show that the plasma response is characterized by the (reduced) plasma wavelength $\lambda_{P}=1 / k_{P}$. In addition there are three scales introduced by the injected laser field: (1) the (reduced) wavelength $\pi=1 / \mathrm{k}=c / \omega,(2)$ the pulse width $w$, and (3) the pulse length $c T_{L}$. The longitudinal, $L_{\|}$, and transverse, $L_{\perp}$, scales appropriate to paraxial propagation are related by [2], [3], $L_{\|}=k L_{\perp}^{2}$. In other words $L_{\|}$is essentially the diffraction length (Rayleigh range) for an aperture of dimension $\mathrm{L}_{\perp}$. For plasmas that are not too underdense $(\omega \mathrm{p} / \omega \geq 0.1)$ and laser pulses that are not too tightly focussed or too short $\left(w / \lambda \approx 10, c T_{L} \approx 10 \lambda\right)$, we may assume that $w / \lambda_{P} \geq O(1)$ and $c T_{L} / \lambda_{p} \geq O(1)$. Then the natural choice for the transverse scale is $\lambda_{p}$, the characteristic transverse size for charge and current inhomogeneities in the plasma, and the longitudinal scale is $\mathrm{L}_{\|}=\mathrm{k} \boldsymbol{\lambda}_{\mathrm{P}}^{2}=\mathrm{k} / \mathrm{k}_{\mathrm{P}}^{2}$. The characteristic opening angle $\theta$ for the paraxial mode,

$$
\theta=\frac{L_{1}}{L_{\|}}=\frac{1}{\sqrt{k L_{\|}}}==\frac{1}{k L_{\perp}}=\frac{k p}{k}=\frac{\omega_{p}}{\omega}
$$

is the small parameter of the theory. Let $n_{c}$ be the critical density at which $\omega_{p}=\omega$, then $\theta=\sqrt{N_{0} / n_{c}}$ which shows that the approximations introduced below are valid for sufficiently underdense plasmas.

I will obtain the slowly varying envelope approximation (SVEA) as the leading order in a multiple scales perturbation theory.[4],[5] This approach provides a quantitative estimate of the errors in the SVEA and also allows for the calculation of higher order effects, such as reflected waves and harmonics of the laser field.

Let $X$ stand for any of the dependent variables, i.e., $X \in\{a, \phi, p, \eta, \gamma\}$, then the first assumption is that $\mathrm{X}$ can be expanded in powers of $\theta$ :

$$
X=\sum_{n=0}^{\infty} \theta^{n} X^{(n)}
$$

Note that this is not an expansion in the strength of the laser field, but rather a high frequency (low density) expansion in powers of $\theta=\omega_{p} / \omega\left(\theta=\sqrt{N_{0} / n_{c}}\right)$. The small parameter $\theta$ will also appear in the argument of $X^{(n)}$ because of the existence of the three very different length scales mentioned above. In this problem there is only one relevant 
transverse scale, the plasma length $L_{\perp}=\lambda_{P}=1 / k_{P}$, but there are three longitudinal scales, $\lambda \ll \lambda_{P} \ll L_{\|}$which are related by powers of $\theta$ :

$$
\lambda=\frac{\lambda_{\mathrm{P}}}{\theta}, \quad \mathrm{L}_{\|}=\frac{\lambda_{\mathrm{P}}}{\theta^{2}},
$$

and three corresponding time scales $\lambda / c<<\lambda_{\mathrm{P}} / c<L_{\mathbb{N}} / \mathrm{c}$, where $\lambda / c=1 / \omega, \lambda_{\mathrm{P}} / \mathrm{c}=1 / \omega_{\mathrm{P}}$, and $L_{\mathbb{V}} / \mathrm{c}=\omega / \omega_{\mathrm{p}}^{2}$, and

$$
\frac{\lambda}{c}=\frac{1}{\theta} \frac{\lambda_{P}}{c}, \frac{L_{\|}}{c}=\frac{1}{\theta^{2}} \frac{\lambda_{P}}{c} .
$$

Plasma excitations are naturally described by the dimensioniess coordinates $r^{*} \equiv k_{p} r$, and $t^{*} \equiv \omega_{P} t$; i.e., $r$ and $t$ should be measured in units of $\lambda_{P}$ and $\lambda_{P} / c$ respectively. The propagative effects require fast variables,

$$
\hat{z}=\frac{z}{\lambda}=k z=\frac{z^{*}}{\theta}, \hat{t}=\frac{c t}{\lambda}=\omega t=\frac{t^{*}}{\theta}
$$

to describe oscillations at optical frequencies, and slow variables

$$
\bar{z}=\frac{z}{L_{\|}}=\theta z^{*}, \bar{t}=\frac{c t}{L_{\|}}=\theta t^{*}
$$

to describe the evolution of the field amplitude. The most general multiple scales ansatz would then be $X^{(n)}=X^{(n)}\left(r^{*}, t^{*}, \bar{z}, \bar{t}, \hat{z}, \hat{t}\right)$, but for the lowest order calculations which neglect reflected waves the fast variable dependence will only involve the combination $\xi \equiv k z-\omega t=\hat{z}-\hat{t}=\left(z^{*}-t^{*}\right) / \theta$, and the appropriate ansatz is

$$
\begin{aligned}
& X^{(n)}=X^{(n)}(r, t, \bar{z}, \bar{t}, \bar{\xi}) \\
& X^{(k)}(r, t, \bar{z}, \bar{t}, \xi)=X_{0}^{(k)}(r, t, \bar{z}, \bar{t})+\frac{1}{2} \sum_{m}^{\prime} X_{m}^{(k)}(r, t, \bar{z}, \bar{t}) e^{i ~ m \xi}, \\
& X_{m}^{(k)}(r, t, \bar{z}, \bar{t})=X_{-m}^{(k) *}(r, t, \bar{z}, \bar{t}),
\end{aligned}
$$

where the *-notation has been dropped $\left(r^{*} \rightarrow r, t^{*} \rightarrow t\right)$, i.e., the general units of length and time are respectively $\lambda_{P}=1 / k_{P}$ and $\lambda_{P} / c=1 / \omega_{P}$. This has the effect of setting 
$\mathrm{k}_{\mathrm{p}}=\mathrm{c}=1$ in (1.14). The scaled variables $r, t, \bar{z}, \bar{t}, \bar{\xi}$ will be treated as formally independent, so the ansatz (2.7a) entails, by a chain rule argument, the replacements

$$
\begin{aligned}
& \frac{\partial}{\partial \mathrm{z}} \rightarrow \frac{1}{\theta} \frac{\partial}{\partial \xi}+\frac{\partial}{\partial \mathrm{z}}+\theta \frac{\partial}{\partial \overline{\mathrm{z}}} \\
& \frac{\partial}{\partial \mathrm{t}} \rightarrow-\frac{1}{\theta} \frac{\partial}{\partial \xi}+\frac{\partial}{\partial \mathrm{t}}+\theta \frac{\partial}{\partial \overline{\mathrm{t}}}
\end{aligned}
$$

The calculation begins by imposing (2.2), (2.7) and (2.8) on the exact equations (1.14) and requiring the expanded equations to be satisfied separately in each order of $\theta$. Thus each of the equations (1.14) will produce a number new equations depending on the maximum expansion order considered. In general the expansion starts with terms of order $\theta^{-2}$ due to the presence of mixed derivatives like $\partial^{2} / \partial z \partial t$; therefore even the zeroth order caiculation will produce as many as three equations for each equation in (1.14). This apparent redundancy is compensated by the extra degrees of freedom introduced by treating the scaled variables $z, t, \bar{z}, \bar{t}, \bar{\zeta}$ as independent. Integration of the new equations will typically produce secular terms, i.e., terms in the higher order coefficients, $X^{(n)}(n>1)$, growing as some power of one of independent time variables $(t, \bar{t}, \bar{\xi})$. The extra degrees of freedom will be used to control the numerical convergence of the $\theta$-series by eliminating secular growth. The ansatz (2.7) allows for the existence of harmonics of the laser field, but a detailed calculation shows that these terms are missing in zeroth order. The reason is that the amplitudes for zeroth order reflected and harmonic terms satisfy homogeneous equations. Since these terms vanish initially they must then vanish at all later times. We have already used this conclusion in the ansatz (2.7a), which excludes any reflected waves, and the absence of harmonics in lowest order allows a further simplification in which the general Fourier expansion (2.7b) is replaced by

$$
X^{(0)}(\mathbf{r}, \bar{z}, \bar{t}, \xi)=X_{w}^{(0)}(r, t, \bar{z}, \bar{t})+\frac{1}{2}\left(X_{L}^{(0)}(r, t, \bar{z}, \bar{t}) e^{i} \xi+C C\right)
$$

where the wake term $\mathrm{X}_{\mathrm{w}}^{(0)}(\mathrm{r}, \mathrm{t}, \overline{\mathrm{z}}, \overrightarrow{\mathrm{t}}) \equiv \mathrm{X}_{0}^{(0)}(\mathrm{r}, \mathrm{t}, \overline{\mathrm{z}}, \overrightarrow{\mathrm{t}})$ is the $(\mathrm{m}=0)$-component of the zeroth-order $\mathrm{X}^{(0)}(\mathbf{r}, \overline{\mathrm{z}}, \overline{\mathrm{t}}, \overline{\mathrm{j}})$ and the laser term $\mathrm{X}_{\mathrm{L}}^{(0)}(\mathrm{r}, \mathrm{t}, \overline{\mathrm{z}}, \overline{\mathrm{t}})=\mathrm{X}_{1}^{(0)}(\mathbf{r}, \mathrm{t}, \overline{\mathrm{z}}, \overline{\mathrm{t}})$ is the $(m=1)$-component. In particular, the zeroth order vector potential and the electron momentum are given by

$$
\mathbf{a}^{(0)}(\mathbf{r}, \overline{\mathbf{z}}, \overline{\mathrm{t}}, \bar{\xi})=\mathbf{a}_{w}^{(0)}(\mathbf{r}, \mathrm{t}, \overline{\mathrm{z}}, \overline{\mathrm{t}})+\frac{1}{2}\left(\mathbf{a}_{\mathrm{L}}^{(0)}(\mathbf{r}, \mathrm{t}, \overline{\mathrm{z}}, \overline{\mathrm{t}}) \mathrm{e}^{\mathrm{i} \xi}+\mathrm{CC}\right)
$$




$$
\mathbf{p}^{(0)}(\mathbf{r}, \bar{z}, \bar{t}, \bar{\xi})=\mathbf{p}_{w}^{(0)}(\mathbf{r}, \mathbf{t}, \bar{z}, \bar{t})+\frac{1}{2}\left(\mathbf{p}_{\mathrm{L}}^{(0)}(\mathbf{r}, \mathbf{t}, \bar{z}, \bar{t}) e^{\mathrm{i} \xi}+C C\right)
$$

where $a_{L}^{(0)}$ is the amplitude of the propagating laser field, $a_{w}^{(0)}$ is the vector potential generated by the charge and current densities produced by the pondermotive force, $p_{L}^{(0)}$ is the quiver momentum, and $\mathbf{p}_{\mathrm{w}}^{(0)}$ is the wake momentum. Since Fourier components with $|m| \geq 2$ are to be neglected, the product of two zeroth order quantities obeys

$$
\begin{aligned}
& X^{(0)} Y^{(0)}=\left[X^{(0)} Y^{(0)}\right]_{w}+\frac{1}{2}\left\{\left[X^{(0)} Y^{(0)}\right]_{L} e^{i \xi}+C C\right\} \\
& {\left[X^{(0)} Y^{(0)}\right]_{w} \equiv X_{w}^{(0)} Y_{w}^{(0)}+\frac{1}{4}\left\{X_{L}^{(0)} Y_{L}^{(0) *}+C C\right\}} \\
& {\left[X^{(0)} Y^{(0)}\right]_{L} \equiv X_{w}^{(0)} Y_{L}^{(0)}+X_{L}^{(0)} Y_{w}^{(0)}}
\end{aligned}
$$

The higher order $X^{(n)}$ 's must be retained in the intermediate stages of the calculation of the $\mathrm{X}^{(0)}$ 's, but they can be dropped at the end.

\section{B. Derivation of the zeroth order equations}

Those not interested in the details of the derivation can skip to Sec. III where the final results for the lowest order will be gathered together. The calculations involved in the argument are straightforward, but rather tedious, so they will be only sketched here.

The relations following from expanding Eqs.(1.14) and prohibiting secularities are listed below.

The gauge condition, Eq.(1.14a):

$$
\begin{aligned}
& \frac{\partial a_{3}^{(0)}}{\partial \xi}=0 \Rightarrow a_{3 L}^{(0)}=0 \\
& \nabla \cdot a_{w}^{(0)}+\frac{1}{2}\left(\nabla \cdot a_{L}^{(0)}(r, t, \bar{z}, \bar{t}) e^{i} \xi+C C\right)+\partial \xi a_{3}^{(1)}=0 .
\end{aligned}
$$

Eq. (2.10a) shows that the zeroth order laser field is purely transverse. Since $\mathbf{a}_{w}^{(0)}$ is independent of $\xi$, integrating (2.10b) will produce a term in $\mathrm{a}_{3}^{(1)}$ linear in $\xi(\xi$-secularity) unless we set

$$
\nabla \cdot \mathbf{a}_{\mathbf{w}}^{(0)}=0
$$


Equivalently, assume that $\mathrm{a}_{3}^{(1)}$ is bo'nded and average $(2.10 \mathrm{~b})$ over $\xi$. The fast terms in (2.10b) determine the first onder correction to the longitudinal component of the laser field:.

$$
a_{3 L}^{(1)}=-i \nabla \cdot g_{L}^{(0)} \text {. }
$$

The wave equation, Eq.(1.14b):

$$
\begin{aligned}
& \frac{\partial^{2} \phi^{(0)}}{\partial \xi^{2}}=0 \Rightarrow \phi L \\
& 2 \frac{\partial}{\partial i} \frac{\partial}{\partial \xi} a^{(0)}=-u_{3} \frac{\partial^{2}}{\partial \xi^{2}} \phi^{(1)}, \\
& 2 \frac{\partial}{\partial \tau} \frac{\partial}{\partial \xi} a^{(0)}=\phi_{w}^{(0)}, \\
& \left.\qquad \nabla^{2}-\frac{\partial^{2}}{\partial t^{2}}+2 \frac{\partial}{\partial \tau} \frac{\partial}{\partial \xi}\right\} a^{(0)}=\eta^{(0)} p^{(0)}+\nabla \partial_{t} \phi_{w}^{(0)}-u_{3} \frac{\partial^{2} \phi^{(2)}}{\partial \xi^{2}},
\end{aligned}
$$

where $u_{3}$ is the unit vector along the $z$-axis, $\tau=t, \zeta=z-t, \bar{\tau}=\bar{t}$, and $\bar{\zeta}=\bar{z}-\bar{t}$. The coordinates $(\zeta, \tau)$ are the "speed-of-light" variables introduced by Sprangle et al [6], measured on the plasma scale, and $(\zeta, \bar{\tau})$ are the same coordinates measured on the longer scale appropriate to the evolution of the field envelope. The transverse components of (2.11b) give

$$
\frac{\partial}{\partial \tau} \mathbf{a}_{\mathbf{L}}^{(0)}=0
$$

and the longitudinal component shows that $\phi_{L}^{(1)}=0$, i.e. $\phi^{(1)}=\phi_{w}^{(1)}$. The condition preventing a $\xi$-secularity in $(2.11 \mathrm{c})$ is obtained by assuming that $\mathrm{a}^{(1)}$ is bounded and $\xi$-averaging:

$$
\left\{\nabla^{2}-\frac{\partial^{2}}{\partial t^{2}}\right\} a_{w}^{(0)}=\left[\eta^{(0)} p(0)\right]_{w}+\nabla \partial_{\mathfrak{t}} \phi_{w}^{(0)} .
$$

This is a wave equation for the wake field in which the slowly varying part of the current acts as the source term. Turning next to the fast terms in $(2.11 \mathrm{c})$, the transverse and longitudinal components are respectively given by 


$$
\begin{aligned}
& 2 \mathrm{i} \frac{\partial}{\partial \tau} a_{L \perp}^{(1)}=-\left\{\nabla^{2}-\frac{\partial^{2}}{\partial t^{2}}+2 \mathrm{i} \frac{\partial}{\partial \bar{\tau}}\right\} a_{L}^{(0)}+\left[\eta^{(0)} p_{\perp}^{(0)}\right]_{L}, \\
& 2 \mathrm{i} \frac{\partial}{\partial \tau} a_{3 L}^{(1)}=\left[\eta^{(0)} p_{3}^{(0)}\right]_{L}-\frac{\partial^{2} \phi^{(2)}}{\partial \xi^{2}} .
\end{aligned}
$$

According to (2.11d) the first term on the right hand side of (2.11f) is independent of $\tau$; consequently, integration in $\tau$ will produce $\tau$-secularities in $\mathrm{a}_{\mathrm{L}}^{(1)}$ which must be prevented by imposing

$$
\left.\left\{\nabla_{\perp}^{2}+2 \mathrm{i} \frac{\partial}{\partial \bar{\tau}}\right\} a_{L}^{(0)}=\sqrt{\left.\eta^{(0)} p_{\perp}^{(0)}\right]_{L}}=\sqrt{\eta_{w}^{(0)} p_{\perp L}^{(0)}+\eta_{L}^{(0)} p_{\perp w}^{(0)}}\right\},
$$

where $\nabla^{2}-\frac{\partial^{2}}{\partial t^{2}}=\nabla_{\perp}^{2}-\frac{\partial^{2}}{\partial \tau^{2}}+2 \frac{\partial}{\partial \tau} \frac{\partial}{\partial \zeta}$, and the right hand side is defined by averaging over the $\tau$-dependence as follows

$$
\bar{X}\left(r_{\perp}, \zeta, \bar{\tau}\right) \equiv \frac{1}{2 T} \int_{-T}^{T} d \tau^{\prime} X\left(r_{\perp}, \zeta, \bar{\tau}+\tau^{\prime}\right)
$$

where $T=O(1 / \theta) \gg>$. This is a paraxial wave equation with the $(m=1)$-part of the current as its source.

Poisson's equation, (1.14c):

$$
\begin{aligned}
& \frac{\partial^{2} \phi_{w}^{(0)}}{\partial \xi^{2}}=0, \quad \frac{\partial^{2} \phi^{(1)}}{\partial \xi^{2}}+2 \frac{\partial}{\partial \xi} \frac{\partial}{\partial z} \phi_{w}^{(0)}=0 \\
& \frac{\partial^{2}}{\partial \xi^{2}} \phi^{(2)}+\nabla^{2} \phi_{w}^{(0)}=\gamma^{(0)} \eta^{(0)}-\mathrm{f} .
\end{aligned}
$$

Eq. (2.12a) is already satisfied by (2.11a-b). Elimination of $\xi$-secularity from (2.12b) gives a Poisson equation for the zeroth order electrostatic potential

$$
\nabla^{2} \phi_{w}^{(0)}=\left[\gamma^{(0)} \eta^{(0)}\right]_{w}-\mathrm{f} .
$$


The Lorentz force law (1.14d):

$$
\begin{aligned}
& -\frac{\partial}{\partial \xi}\left(p^{(0)}-\mathbf{a}^{(0)}\right)=\mathbf{u}_{3} \frac{\partial}{\partial \xi}\left(\phi_{w}^{(0)}-\gamma^{(0)}\right)=-\mathbf{u}_{3} \frac{\partial}{\partial \xi} \gamma^{(0)}, \\
& \frac{\partial}{\partial t}\left(\mathbf{p}^{(0)}-\mathbf{a}(0)\right)-\frac{\partial}{\partial \xi}\left(\mathbf{p}^{(1)}-\mathbf{a}^{(1)}\right)=\nabla\left(\phi_{w}^{(0)}-\gamma^{(0)}\right) .
\end{aligned}
$$

The transverse and longitudinal parts of (2.13a) yield

$$
p_{\perp L}^{(0)}=a_{L}^{(0)}, \quad p_{3 L}^{(0)}=\gamma_{L}^{(0)},
$$

where the first equation is the familiar statement that the quiver momentum is proportional to the vector potential of the laser field, and the second relates the $(m=1)$-(quiver) parts of the longitudinal momentum and the energy. A more detailed calculation shows that the second equation generalizes to $\mathrm{p}_{3 \mathrm{f}}^{(0)}=\gamma_{\mathrm{f}}^{(0)}$, where $\mathrm{X}_{\mathrm{f}}^{(0)} \equiv \mathrm{X}^{(0)}-\mathrm{X}_{0}^{(0)}$ includes the entire $\xi$-dependence of $\mathrm{X}^{(0)}$, not just the $(\mathrm{m}=1)$-term. Elimination of $\xi$-secularity from $(2.13 \mathrm{~b})$ yields the equation of motion for the wake momentum.

$$
\frac{\partial}{\partial t}\left(\mathbf{p}_{w}^{(0)}-\mathbf{a}_{w}^{(0)}\right)=\nabla\left(\phi_{w}^{(0)}-\gamma_{w}^{(0)}\right) .
$$

The remaining rapidly varying terms in (2.13b) determine first order corrections, but do not lead to any secular behavior.

The continuity equation (1.14e):

$$
\begin{aligned}
& \partial_{\xi}\left[\eta^{(0)}\left(\mathrm{p}_{3}^{(0)}-\gamma^{(0)}\right)\right]=0, \\
& \partial_{\xi}\left[\eta^{(1)}\left(\mathrm{p}_{3}^{(0)}-\gamma^{(0)}\right)+\eta^{(0)}\left(\mathrm{p}_{3}^{(1)}-\gamma^{(1)}\right)\right]+\partial_{\mathrm{t}}\left(\eta^{(0)} \gamma^{(0)}\right)+\nabla \cdot\left(\eta^{(0)} \mathrm{p}^{(0)}\right)=0 .
\end{aligned}
$$

Eq.(2.14a), together with (2.13c), yields

$$
\left(\gamma_{w}^{(0)}-p_{3 w}^{(0)}\right) \eta_{L}^{(0)}=0
$$

One solution of $(2.14 c)$, is $\left(\gamma_{w}^{(0)}-p_{3 w}^{(0)}\right)=0$, but in combination with $(2.13 c)$ this would imply $\gamma^{(0)}=p_{3}^{(0)}$. This is inconsistent with $\gamma=\sqrt{1+p^{2}}$ which, in zeroth order yields 


$$
\gamma^{(0)}=\sqrt{1+\left(p^{(0)}\right)^{2}}=\sqrt{1+\left(p_{\perp}^{(0)}\right)^{2}+\left(p_{3}^{(0)}\right)^{2}}>\left|p_{3}^{(0)}\right|
$$

Therefore, the only permissible solution for $(2.14 \mathrm{c})$ is

$$
\eta_{\mathrm{L}}^{(0)}=0
$$

that is, the zeroth order electron density has no rapidly varying part.

Elimination of $\xi$-secularity from (2.14b) produces a continuity equation for the slowly varying wake quantities

$\partial_{l}\left(\eta_{w}^{(0)} \gamma_{w}^{(0)}\right)+\nabla \cdot\left(\eta_{w}^{(0)} p_{w}^{(0)}\right)=0$

The remaining rapidly varying terms do not lead to any secular behavior in higher orders, so they need not be considered for the zeroth order calculation.

The results obtained above allow some simplifications. Thus $(2.14 \mathrm{e})$ together with (2.13c) implies

$$
\overline{\left.\eta^{(0)} \mathbf{p}_{\perp}^{(0)}\right]_{L}}=\overline{\eta_{w}^{(0)} \mathbf{p}_{\perp L}^{(0)}}=\overline{\eta_{w}^{(0)}} \mathbf{a}_{L}^{(0)}
$$

where the final step uses the fact, c.f. $(2.11 \mathrm{~d})$, that $\mathrm{a}_{\mathrm{L}}^{(0)}$ is independent of $\tau$. This simplifies (2.11h) to

$$
\left\{\nabla^{2}-\frac{\partial^{2}}{\partial t^{2}}+2 i \frac{\partial}{\partial \bar{\tau}}\right\} a_{L}^{(0)}=\bar{\eta}_{w}^{(0)} a_{L}^{(0)} .
$$

Some surprising consequences follow for $\gamma^{(0)}$ :

$$
\begin{aligned}
\gamma^{(0)} & =\sqrt{1+\left(p^{(0))^{2}}\right.}=\sqrt{1+\left(p_{\perp}^{(0)}\right)^{2}+\left(p_{3}^{(0)}\right)^{2}} \\
& =\sqrt{1+\left(p_{\perp w}^{(0)}+p_{\perp f}^{(0)}\right)^{2}+\left(p_{3 w}^{(0)}+p_{3 f}^{(0)}\right)^{2}}
\end{aligned} .
$$

Use $(2.13 c)\left(\mathbf{p}_{\perp f}^{(0)}=\mathbf{a}_{\perp f}^{(0)}\right.$ and $\left.\gamma_{f}^{(0)}=p_{3 f}^{(0)}\right)$ to reexpress this as

$$
\gamma^{(0)}=\sqrt{1+\left(\mathbf{p}_{w}^{(0)}\right)^{2}+2 \mathbf{p}_{\perp w}^{(0)} \cdot \mathbf{a}_{\perp f}^{(0)}+\left(\mathbf{a}_{\perp f}^{(0)}\right)^{2}+2 p_{3 w}^{(0)} \gamma_{f}^{(0)}+\left(\gamma_{f}^{(0)}\right)^{2}},
$$


then square both sides and use $\gamma^{(0)}=\gamma_{w}^{(0)}+\gamma_{f}^{(0)}$ to get

$$
\left(\gamma_{w}^{(0)}\right)^{2}+2 \gamma_{w}^{(0)} \gamma_{f}^{(0)}=1+\left(p_{w}^{(0)}\right)^{2}+2 p_{\perp w}^{(0)} \cdot a_{\perp f}^{(0)}+\left(a_{\perp f}^{(0)}\right)^{2}+2 p_{3 w}^{(0)} \gamma_{f}^{(0)}
$$

The $\xi$-average of this yields

$$
\left(\gamma_{w}^{(0)}\right)^{2}=1+\left(\mathbf{p}_{w}^{(0)}\right)^{2}+\left[\left(\mathbf{a}_{\perp f}^{(0)}\right)^{2}\right]_{w} \Rightarrow \gamma_{w}^{(0)}=\sqrt{1+\left(\mathbf{p}_{w}^{(0)}\right)^{2}+\left[\left(\mathbf{a}_{\perp f}^{(0)}\right)^{2}\right]_{w}},
$$

ie., the $\xi$-average of $\gamma^{(0)}$ can be calculated by replacing the argument of the square root in (2.17a) by its $\xi$-average! The final expression comes from

$$
\begin{aligned}
& \mathbf{a}_{\perp f}^{(0)}=\frac{1}{2}\left\{\mathbf{a}_{\perp \perp}^{(0)} \mathrm{e}^{\mathrm{i} \xi}+c c\right\} \Rightarrow\left[\left(\mathbf{a}_{\perp f}^{(0)}\right)^{2}\right]_{w}=\frac{1}{2}\left|\mathrm{a}_{\perp \perp}^{(0)}\right|^{2} \Rightarrow \\
& \gamma_{w}^{(0)}=\sqrt{1+\left(\mathbf{p}_{w}^{(0)}\right)^{2}+\frac{1}{2}\left|a_{L}^{(0)}\right|^{2}} .
\end{aligned}
$$

The $\xi$-dependence of $\gamma^{(0)}$ is then determined by $(2.17 \mathrm{~b}, \mathrm{~d})$

$$
\begin{aligned}
& 2\left(\gamma_{0}^{(0)}-2 p_{30}^{(0)}\right) \gamma_{f}^{(0)}=2 p_{\perp 0}^{(0)} \cdot a_{\perp f}^{(0)}+\left[\left(a_{\perp f}^{(0)}\right)^{2}\right]_{f}, \\
& {\left[\left(a_{\perp f}^{(0)}\right)^{2}\right]_{f}=\frac{1}{4}\left[\left(a_{L}^{(0)}\right)^{2} e^{2 i \xi}+c c\right] \Rightarrow} \\
& \gamma_{L}^{(0)}=\gamma_{1}^{(0)}=\frac{p_{\perp w}^{(0)} \cdot a_{L}^{(0)}}{\left(\gamma_{w}^{(0)}-2 p_{3 w}^{(0)}\right)}=p_{3 L}^{(0)}, \\
& \gamma_{2}^{(0)}=\frac{\left(a_{L}^{(0)}\right)^{2}}{4\left(\gamma_{w}^{(0)}-2 p_{3 w}^{(0)}\right)}=\left(p_{3,2}^{(0)}\right), \\
& \gamma_{m}^{(0)}=0=p_{3, m}^{(0)}, \forall|m| \geq 2 .
\end{aligned}
$$

Note that the proper density $\eta$ has no $\xi$-dependence, i.e., it does not oscillate on the optical scale, but the density $\rho=\eta \gamma$ (measured in the laboratory frame) does have such dependence:

$$
\rho^{(0)}=\eta_{w}^{(0)}\left\{\gamma_{w}^{(0)}+\frac{1}{2}\left(\gamma_{L}^{(0)} \mathrm{e}^{\mathrm{i} \xi}+C C\right)+\frac{1}{2}\left(\gamma_{2}^{(0)} \mathrm{e}^{2 \mathrm{i} \xi}+C C\right)\right\}
$$

Page 14 


\section{Zeroth order theory}

\section{A Radiation-gauge equations}

The notation can be considerably simplified by using the results of the last section. Since no higher order terms are needed, the superscript (0) can be dropped. Also, the proper charge density $\eta^{(0)}$ and the scalar potential $\phi^{(0)}$ have no fast dependence so the subscript $w$ can be omitted, $\eta_{w}^{(0)} \rightarrow \eta, \phi_{w}^{(0)} \rightarrow \phi$. Only the slowly varying part of the energy $\gamma_{w}^{(0)}$ will play a role so I set $\gamma_{w}^{(0)} \rightarrow \gamma$. The zeroth order equations in this new notation are

$$
\begin{aligned}
& \left\{\nabla_{\perp}^{2}+2 i\left(\frac{\partial}{\partial \bar{z}}+\frac{\partial}{\partial \bar{t}}\right)\right\} a_{L}=\bar{\eta} a_{L}, \\
& \left(\frac{\partial}{\partial z}+\frac{\partial}{\partial t}\right) a_{L}=\frac{\partial}{\partial \tau} a_{L}=0, \\
& \nabla \cdot a_{w}=0, \\
& \left\{\nabla^{2}-\frac{\partial^{2}}{\partial t^{2}}\right\} \mathbf{a}_{w}=\eta p_{w}+\nabla \partial_{t} \phi, \\
& \nabla^{2} \phi=\gamma \eta \eta f, \\
& \frac{\partial}{\partial t}\left(\mathbf{p}_{w}-\mathbf{a}_{w}\right)=\nabla(\phi-\gamma), \\
& \frac{\partial}{\partial t}(\eta \gamma)+\nabla \cdot\left(\eta p_{w}\right)=0, \\
& \gamma=\sqrt{1+\left(\mathbf{p}_{w}\right)^{2}+\frac{1}{2}\left|a_{L}\right|^{2}}, \\
& \bar{z}=\theta z, \bar{t}=\theta t, \\
& \bar{\eta}\left(\mathbf{r}_{\perp}, \zeta, \bar{\tau}\right) \equiv \frac{1}{2 T} \int_{-\tau}^{T} d \tau^{\prime} \eta\left(\mathbf{r}_{\perp}, \zeta, \bar{\tau}+\tau^{\prime}\right),
\end{aligned}
$$


$\zeta=\mathbf{z}-\mathrm{t}, \tau=\mathbf{t}$

\section{B Lorentz-gauge equations}

The analysis presented in Secs I and II described the electromagnetic field in the radiation gauge. The same analysis applied to the Lorentz gauge produces the following physically equivalent model:

$$
\begin{aligned}
& p_{L \perp}=a_{L \perp}, p_{L 3}=\gamma_{L}, a_{L 3}=\phi_{L}, \eta_{L}=0 \Rightarrow \eta=\eta_{w}, \\
& \left\langle\nabla_{\perp}^{2}+2 \mathrm{i}\left(\frac{\partial}{\partial \bar{z}}+\frac{\partial}{\partial \bar{t}}\right)\right\} a_{L \perp}=\bar{\eta} a_{L \perp}, \\
& \left\{\nabla_{\perp}^{2}+2 i\left(\frac{\partial}{\partial \bar{z}}+\frac{\partial}{\partial \bar{t}}\right)\right\} a_{L 3}=\overline{\left(\frac{\eta p_{w \perp} \cdot a_{L \perp}}{\gamma_{w}-p_{3 w}}\right)} \\
& \left(\frac{\partial}{\partial z}+\frac{\partial}{\partial t}\right) \mathbf{a}_{L}=\frac{\partial}{\partial \tau} \mathbf{a}_{L}=0, \quad\left(a_{L}(r, t, \bar{z}, \bar{t})=a_{L}\left(\mathbf{r}_{\perp}, z-t, \bar{z}, \bar{t}\right)\right), \\
& \nabla \cdot \mathbf{a}_{w}+\frac{\partial \phi_{w}}{\partial t}=0 \text { (Lorentz condition for wake field) } \\
& \left\{\nabla^{2}-\frac{\partial^{2}}{\partial t^{2}}\right\} \mathbf{a}_{w}=\eta p_{w} \\
& \left\{\nabla^{2}-\frac{\partial^{2}}{\partial t^{2}}\right\} \phi_{w}=\gamma_{w} \eta-f \\
& \frac{\partial}{\partial t}\left(\mathbf{p}_{w}-\mathbf{a}_{w}\right)=\nabla\left(\phi_{w}-\gamma_{w}\right) \\
& \partial_{t}\left(\eta \gamma_{w}\right)+\nabla \cdot\left(\eta p_{w}\right)=0 \\
& \gamma_{w}=\sqrt{1+\left(\mathbf{p}_{w}\right)^{2}+\frac{1}{2}\left|\mathbf{a}_{L}\right|^{2}} \\
& \gamma_{L}=\frac{\mathbf{p}_{w \perp} \cdot \mathbf{a}_{L \perp}}{\gamma_{w}-p_{3 w}}
\end{aligned}
$$




$$
\bar{\eta}\left(\mathbf{r}_{\perp}, \zeta, \bar{\tau}\right) \equiv \frac{1}{2 \mathrm{~T}} \int_{-\mathrm{T}}^{\mathrm{T}} \mathrm{d} \tau^{\prime} \eta\left(\mathbf{r}_{\perp}, \zeta, \bar{\tau}+\tau^{\prime}\right), \zeta=\mathrm{z}-\mathrm{t}, \tau=\mathrm{t}
$$

In this gauge, the vector potential for the laser has a nonvanishing $z$-component and the scalar potential has a rapidly varying part. These quantities are related by (3.9) which replaces the transversality condition of the radiation gauge. Eq.(3.10b) for the $z$-component of the laser field is redundant since aL3 does not enter into the remaining equations. Note that the wake scalar potential $\phi_{w}$ is not the electrostatic potential since it satisfies the wave equation rather than Poisson's equation. For numerical computations it is convenient to eliminate the slow variables $\overline{\mathrm{z}}$ and $\overline{\mathrm{t}}$ by the definition (2.6). At the same time the averaging over $\tau$ and the constraint relation (3.11) can be dropped. The resulting equations are

$$
\begin{aligned}
& \left\{\nabla_{\perp}^{2}+\frac{2 i}{\theta}\left(\frac{\partial}{\partial z}+\frac{\partial}{\partial t}\right)\right\} a_{L \perp}=\eta a_{L \perp}, \\
& \nabla \cdot \mathbf{a}_{w}+\frac{\partial \phi_{w}}{\partial t}=0, \\
& \left\{\nabla^{2}-\frac{\partial^{2}}{\partial t^{2}}\right\} \mathbf{a}_{w}=\eta p_{w}, \\
& \left\{\nabla^{2}-\frac{\partial^{2}}{\partial t^{2}}\right\} \phi_{w}=\gamma_{w} \eta-f \\
& \frac{\partial}{\partial t}\left(\mathbf{p}_{w}-\mathbf{a}_{w}\right)=\nabla\left(\phi_{w}-\gamma_{w}\right), \\
& \partial_{t}\left(\eta \gamma_{w}\right)+\nabla \cdot\left(\eta \mathbf{p}_{w}\right)=0, \\
& \gamma_{w}=\sqrt{1+\left(\mathbf{p}_{w}\right)^{2}+\frac{1}{2}\left|\mathbf{a}_{L \perp}\right|^{2}} .
\end{aligned}
$$

Eq. (3.20) follows from the rest if imposed initially 


\section{Discussion of previous work}

I first consider the work of Sun et al [7] which provides the basis for the later work of Borisov et al [8]. These authors use a multiple scales technique, but the initial ansatz differs crucially from the one used in Sec. II. In my notation, their ansatz is

$$
X^{(n)}=X^{(n)}(\mathbf{r}, \bar{z}, \xi),
$$

i.e., the only time dependence allowed is in the fast variable $\xi$. Under this strong assumption the only time dependent plasma response allowed is the quiver motion. Sun $e t$ al use the Lorentz gauge so the effects of their ansatz can be seen by dropping all $t-$ and $\bar{t}$-derivatives in the Lorentz-gauge equations (3.9)-(3.17). Since the wake momentum $\mathbf{p}_{\mathbf{w}}$ and vector potential $a_{w}$ vanish initially, they must vanish identically at all times. The equations that survive this ansatz are

$$
\begin{aligned}
& \left\{\nabla_{\perp}^{2}+2 i \frac{\partial}{\partial \bar{z}}\right\} a_{L \perp}=\eta a_{L \perp} \\
& \nabla^{2} \phi_{w}=\gamma_{w} \eta-f \\
& \nabla\left(\phi_{w}-\gamma_{w}\right)=0 \\
& \gamma_{w}=\sqrt{1+\frac{1}{2}\left|a_{L \perp}\right|^{2}}
\end{aligned}
$$

Thus the laser field $a_{L \perp}$ satisfies a stationary paraxial equation, $\phi_{w}$ is the electrostatic potential, and (4.4) is the condition for equilibrium between the Coulomb and pondermotive forces.

An alternative way of obtaining this model is to average Eqs.(3.9)-(3.17) over $t$ and $\bar{t}$. For example, consider the average of the time-dependent paraxial equation (3.10a):

$$
\left\langle\nabla_{\perp}^{2}+2 \mathrm{i} \frac{\partial}{\partial \bar{z}}\right\}\left\langle\mathrm{a}_{L \perp}\right\rangle=\left\langle\eta \mathrm{a}_{L \perp}\right\rangle
$$

where $\left\{. . \cdot\right.$ indicates the average over $t$ and $\bar{t}$. If the fluctuations $a_{L_{\mathcal{L}}}-\left\langle\mathbf{a}_{L_{1}}\right\rangle$ and $\eta-\langle\eta\rangle$ are assumed to be negligible, then (4.6) is approximately the same as 


$$
\left\langle\nabla_{\perp}^{2}+2 \mathrm{i} \frac{\partial}{\partial \overline{\mathbf{z}}}\right\}\left\langle\mathbf{a}_{L \perp}\right\rangle=\langle\eta\rangle\left\langle\mathbf{a}_{L \perp}\right\rangle
$$

which has the form of (4.2). The other equations can be obtained by the same argument. The range of validity of this approximation is not clear. The fully time dependent equations (3.9)-(3.17) will produce nonzero values of $\mathbf{p}_{w}$ and $\mathbf{a}_{\mathbf{w}}$ despite the fact that they vanish initially. The time-averaged treatment would be a good approximation if the time dependent solution somehow approaches the steady state solution with a relatively short relaxation time, but no dissipative mechanism is present in the model which could drive this relaxation. Another possibility is that the wake quantities $\mathbf{p}_{\mathbf{w}}$ and $\mathbf{a}_{\mathbf{w}}$ oscillate around their average values from the beginning. This must be tested by calculations in specific cases. These difficulties do not prevent the exact derivation of the equilibrium condition (4.4), since averaging the linear equation (3.23) over $t$ produces

$$
\nabla\left\langle\phi_{w}\right\rangle=\nabla\left\langle\gamma_{w}\right\rangle,
$$

without any further assumptions. In other words, the equilibrium between electrostatic and pondermotive forces is valid when averaged over many plasma periods.

Next I turn to the work of Sprangle et al $[6,9]$ which is formulated in the radiation gauge. They analyze the problem in the speed-of-light variables introduced in Sec. II. In my notation these are $\tau=t, \zeta=z-t, \bar{\tau}=\bar{t}$, and $\bar{\zeta}=\bar{z}-\bar{t}$. The radiation gauge equations in these coordinates are

$$
\begin{aligned}
& \left\{\nabla_{\perp}^{2}+2 i \frac{\partial}{\partial \bar{\tau}}\right\} \mathbf{a}_{L}=\bar{\eta} \mathbf{a}_{L}, \\
& \frac{\partial}{\partial \tau} \mathbf{a}_{L}=0, \\
& \nabla \cdot \mathbf{a}_{w}=0, \\
& \left\{\nabla_{\perp}{ }^{2}+2 \frac{\partial}{\partial \tau} \frac{\partial}{\partial \zeta}-\left(\frac{\partial}{\partial \tau}\right)^{2}\right\} \mathbf{a}_{w}=\eta \mathbf{p}_{w}-\nabla \frac{\partial}{\partial \zeta} \phi_{w}+\nabla \frac{\partial}{\partial \tau} \phi_{w}, \\
& \nabla^{2} \phi_{w}=\gamma_{w} \eta-f, \\
& \left(\frac{\partial}{\partial \tau}-\frac{\partial}{\partial \zeta}\right)\left(\mathbf{p}_{w}-\mathbf{a}_{w}\right)=\nabla\left(\phi_{w}-\gamma_{w}\right),
\end{aligned}
$$




$$
\begin{aligned}
& \left(\frac{\partial}{\partial \tau}-\frac{\partial}{\partial \zeta}\right)\left(\eta \gamma_{w}\right)+\nabla \cdot\left(\eta p_{w}\right)=0, \\
& \gamma_{w}=\sqrt{1+p_{w}^{2}+\left.\frac{1}{2}\right|_{L} ^{2}{ }^{2}} .
\end{aligned}
$$

The first thing to note here is that $\mathbf{a}_{\mathrm{L}}$ is independent of $\tau, c f(4.10)$. The wake equations (4.11)-(4.16) consequently have a $\tau$-independent solution. When does this solution represent the actual behavior of the plasma? Sprangle et al argue that this will be the case when the distance traversed by a relativistic electron during the pulse duration is small compared to the diffraction length $L_{\|\|}$, i.e., (in conventional units) $c T_{L} \ll L_{\|}$. The idea is that $\sigma \equiv c T_{V} L_{\| l} \ll 1$ means that the plasma does not have suffirient time to respond during the pulse. They then propose a quasi-static approximation in which all $\tau$-derivatives are dropped. There are two difficulties with this argument. First, the $\tau$-stationary solution of the wake equations is inconsistent with the initial conditions, $\mathbf{a}_{\mathrm{L}}=\mathbf{p}_{\mathbf{w}}=0$. Thus the $\tau$-evolving solution would have to relax to the $\tau$-stationary solution in a time short compared to the pulse duration. It is not clear that any mechanism exists to bring this about. Second, the parameter $\sigma$ can be expressed as

$$
\sigma \equiv \frac{c T_{L}}{L_{\|}}=\frac{c T_{L}}{k k_{P}^{-2}}=\frac{k_{P}}{k} \omega_{P} T_{L}=\theta \varepsilon
$$

where $\varepsilon \equiv \omega_{\mathrm{p}} \mathrm{T}_{\mathrm{L}}$. For underdense plasmas $\theta<1$, so $\sigma$ can be small even for moderately large values of $\varepsilon$. On the other hand $\varepsilon>1$ means that the pulse duration includes several plasma periods, so that several plasma oscillations can occur during the pulse. Thus the appropriate condition for no dynamic response would seem to be $\varepsilon=\omega_{\mathrm{p}} T_{\mathrm{L}} \ll 1$. Note that the distance traversed by an electron during one plasma period is $c / \omega_{\mathrm{P}}=1 / \mathrm{kP}_{\mathrm{P}}$ and that

$$
\frac{k_{p}^{-1}}{L_{\|}}=\frac{k_{p}^{-1}}{k k_{p}^{-2}}=\frac{k_{p}}{k}=\theta
$$

That is, the ratio of distance traversed during one plasma period to the diffraction length is small for underdense plasmas; nevertheless the plasma can still respond if $\varepsilon=\omega_{p} T_{L} \gtrsim 1$. In view of these difficulties it would seem prudent to attempt the solution of the full $\tau$-dependent equations (4.9)-(4.16) or the equivalent forms (3.1)-(3.8). 


\section{Critical power}

The laser intensity required to produce phenomena like self focussing and cavitation is an important issue for experimental planning. This value is usually reported in terms of the total power in the laser beam.[7],[8] Reverting for the moment to conventional units, th: laser power is

$$
\widetilde{P}(z, t)=\int d^{2} r_{\perp} I(r, t)=\frac{c}{4 \pi} \int d^{2} r_{\perp} \overline{E_{L}^{2}(r, t)}
$$

where $I$ is the laser intensity, and the overbar again indicates an average over many optical periods. After converting to the normalized variables $\left(d^{2} r_{\perp} \rightarrow k_{P}^{-2} d^{2} r_{\perp}, A_{L} \rightarrow\left(\frac{m c^{2}}{e}\right) a_{L}\right)$ and using (2.9b) to evaluate the laser field, this becomes

$$
\begin{aligned}
\widetilde{P}(z, t) & =\frac{c}{8 \pi}\left(\frac{m c^{2}}{e}\right)^{2}\left(\frac{k}{k_{P}}\right)^{2} \int d^{2} r_{\perp} \mid a_{\perp}\left(r_{\perp}, z-t, z, \bar{t}\right)^{2} \\
& =P(\zeta, \bar{\zeta})\left(\frac{k}{k_{P}}\right)^{2}\left(2.18 \times 10^{9} \mathrm{~W}\right) .
\end{aligned}
$$

The critical power will therefore depend on the numerical value of the dimensionless power

$$
\mathrm{P}(\zeta, \bar{\zeta})=\left.\int \frac{\mathrm{d}^{2} \mathrm{r}_{\perp}}{2 \pi}\left|\mathrm{a}_{\mathrm{L}} \mathrm{P}^{2}=\int_{0}^{\infty} \rho \mathrm{d} \rho\right| \mathrm{a}_{\mathrm{L}}\right|^{2},
$$

where the last form is correct when the field has cylindrical symmetry. The power only depends on $\zeta$ and $\bar{\zeta}$ by virtue of (3.1b) and the conservation law

$$
\left(\frac{\partial}{\partial \bar{z}}+\frac{\partial}{\partial \bar{t}}\right) P(\bar{\zeta}, \bar{\tau})=\frac{\partial}{\partial \bar{\tau}} P(\bar{\zeta}, \bar{\tau})=0,
$$

which is derived from the paraxial wave equation (3.1a).

With the normalizations chosen here, we can expect that the critical dimensionless power $\mathrm{P}_{\mathrm{c}}=O(1)$, but the exact value can only be obtained by a numerical calculation, $c . f$., e.g.[7]. 


\section{Summary and discussion}

The arguments presented in Sec. II yield the full consequences of two assumptions: (1) an underdense plasma $\left(\omega_{\mathrm{p}}<<\omega\right)$, (2) paraxial propagation of the laser beam. This leads to the equations (3.1)-(3.8), in the radiation gauge, or (3.9)-(3.17), in the Lorentz gauge. In Sec. IV I used this analysis in a critical examination of the work of Sun et al [7] and Sprangle $e t$ al $[6,9]$. The multiple-scales ansatz used by Sun et al unnecessarily restricts the temporal dependence of the plasma variables and thereby eliminates consideration of wake phenomena which may well be important. These wake effects are included in the treatment of Sprangle et al , but the quasi-static approximation they employ is not consistent with the initial values of the wake fields, and it is based on a criterion of small ballistic motion of electrons which does not properly take into account plasma oscillations. The scaling analysis also shows that the critical power for cavitation must depend on the ratio $\omega_{\mathrm{p}} / \omega$ according to (5.2) with a dimensionless coefficient of order unity that has to be determined by numerical calculation. From these considerations it is clear that the next step in this program should be a numerical calculation using the full time dependent equations (3.1)-(3.8) \{or (3.9)-(3.17)\}. 


\section{References}

1. Jackson, J.D., Classical Electrodynamics. 2nd ed. 1975, New York: John Wiley \& Sons, Inc. ,p. 221

2. Lax, M., Louisell, W. H.and McKnight, W. B., Phys. Rev. A, 1974. 11: p. 1365.

3. Deutsch, I.H.and Garrison, J. C., Phys. Rev. A, 1991. 43: p. 2498.

4. Wong, J., Garrison, J. C. and Einwohner, T. H., Phys. Rev. A, 1976. 13: p. 674.

5. Sandri, G., Ann. Phys. NY, 1963. 24: p. 380.

6. Sprangle, P., Esarey, E., Krall, J. and Joyce, G., Phys. Rev. Lett., 1992. 69: p. 2200.

7. Sun, G.-Z., Ott, E., Lee, Y. C., and Guzdar, P.,Phys. Fluids, 1987. 30: p. 526-532.

8. Borisov, A.B., Borovskiy, A. V.,Shiryaev, O. B., Solem, J. C., Luk, T. S.,Boyer, K. and Rhodes, C. K., Phys. Rev. A, 1992. 45: p. 5830-5845.

9. Sprangle, P., Esarey, E., and Ting, A., Phys. Rev. Lett, 1990. 64: p. 2011-2014. 

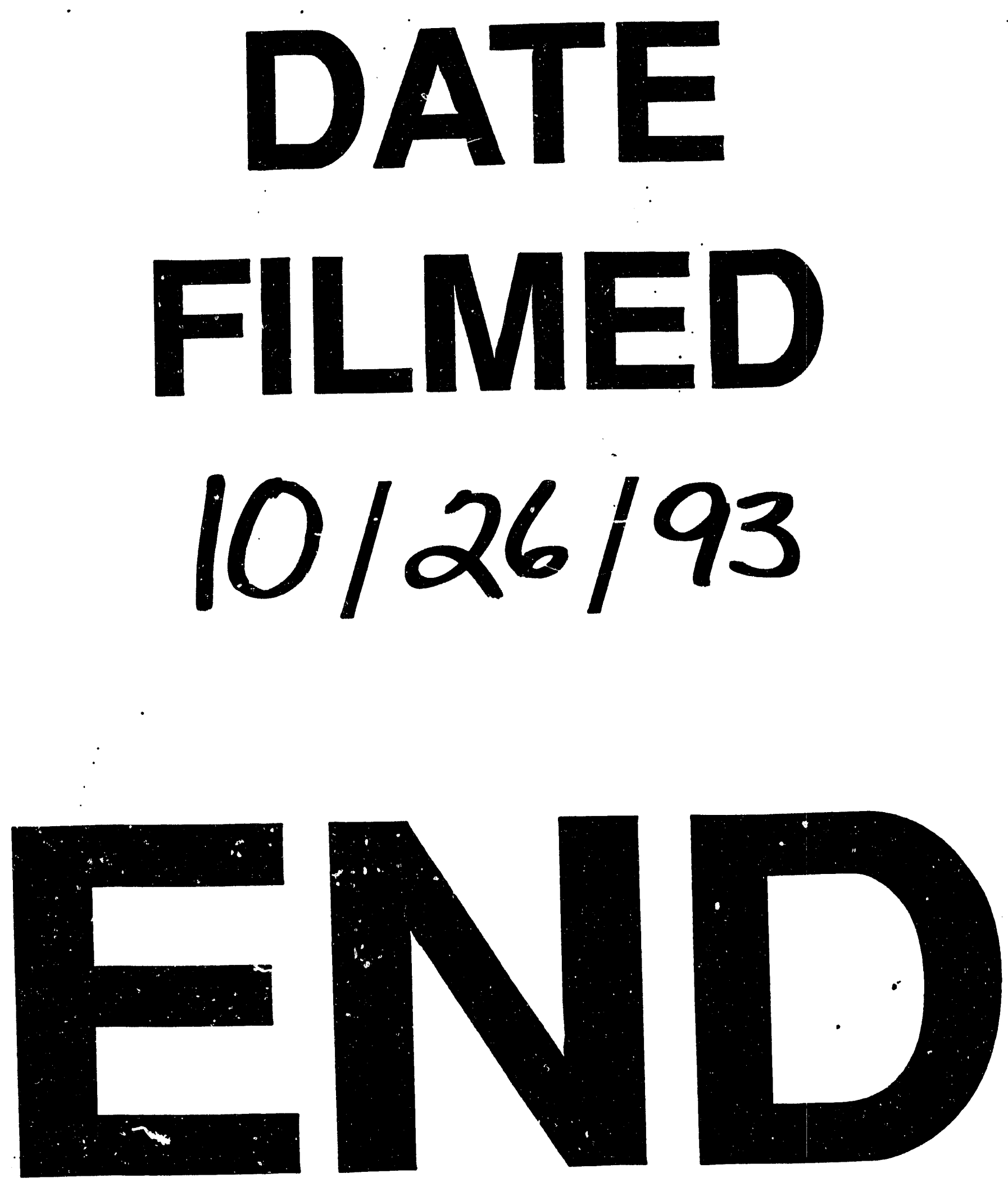
